\title{
Surgical conversion after thoracic endovascular aortic repair
}

\author{
Ludovic Canaud, MD, Pierre Alric, MD, PhD, Thomas Gandet, MD, Bernard Albat, MD, PhD, \\ Charles Marty-Ané, MD, PhD, and Jean-Philippe Berthet, MD
}

\begin{abstract}
Objective: Improved early and late outcomes of thoracic endovascular aortic repair compared with open repair have changed the therapeutic paradigm of thoracic aortic lesions. However, rare but serious complications due to device failure or adverse events may occur, requiring conversion to open repair.
\end{abstract}

\begin{abstract}
Methods: In our experience, 186 patients underwent thoracic endovascular aortic repair. Seven of these patients $(3.7 \%)$ required open repair because of 3 retrograde type A dissections, 1 thoracic stent-graft collapse, 1 aneurysm enlargement without endoleak, 1 aortoesophageal fistula, and 1 stent-graft infection. All patients but 1 underwent surgical repair using cardiopulmonary bypass. Four stent-grafts were totally removed, and 3 endografts were left in situ. Three patients underwent supracoronary ascending aorta replacement via a sternotomy. Three patients underwent a descending aortic replacement via a left thoracotomy. One patient was treated by ligation of the aortic arch, ascending to supraceliac abdominal aorta bypass, and stent-graft explantation.
\end{abstract}

Results: Thirty-day mortality was $28 \%$. Four patients had an uneventful postoperative course. One patient was treated for postoperative sternitis. Two patients with stent-graft infections died of multiorgan failure in the early postoperative course. No stroke, paraplegia, or renal failure occurred. With a mean follow-up of 21.4 months (range, 2-60 months), 5 patients had no adverse events.

Conclusions: Complications due to device failure or adverse events may occur after thoracic endovascular aortic repair, requiring conversion to open repair. Our experience suggests that in some clinical or anatomic situations, caution should be recommended when offering endovascular procedures to patients with thoracic aortic diseases. Open conversion can be performed with encouraging results by a team experienced in the management of thoracic aortic diseases. With the increasing use of thoracic endovascular aortic repair, more patients will present with indications of surgical conversion. (J Thorac Cardiovasc Surg 2011;142:1027-31)

Thoracic endovascular aortic aneurysm repair (TEVAR) is associated with improved early and late outcomes compared with open repair, including lower perioperative morbidity and mortality. This difference in outcome has resulted in TEVAR replacing open repair as the procedure of choice for most patients with thoracic aortic aneurysms and suitable anatomy. However, concerns over long-term endograft durability remain. Reintervention after TEVAR is reported in up to $3.8 \%$ of patients independently of the type of endograft implanted. ${ }^{1}$ Lifelong surveillance is recommended to identify potential late complications of device failure, device infection, endoleak, and endograft migration that may lead to aneurysm rupture.

Late surgical conversion may be an emergency procedure for aneurysm rupture, retrograde type A dissection (RTAD), and graft infection, or an elective procedure for aneurysm enlargement in the presence of an endoleak or endograft mi-

\footnotetext{
From the Department of Thoracic and Cardiovascular Surgery, Arnaud de Villeneuve Hospital, Montpellier, France.

Disclosures: Authors have nothing to disclose with regard to commercial support.

Received for publication Oct 23, 2010; revisions received Jan 6, 2011; accepted for publication Jan 25, 2011; available ahead of print March 14, 2011.

Address for reprints: Ludovic Canaud, MD, Service de Chirurgie Vasculaire et Thoracique, Hôpital A de, Villeneuve, 191 av Doyen Gaston Giraud 34090, Montpellier,

France (E-mail: ludoviccanaud@hotmail.com).

$0022-5223 / \$ 36.00$

Copyright (c) 2011 by The American Association for Thoracic Surgery

doi:10.1016/j.jtcvs.2011.01.060
}

gration not amenable to endovascular interventions. The presence of the endograft complicates surgical exposure, often resulting in higher operative morbidity and mortality compared with primary open repair.

We report our surgical management in 7 patients who required conversion to open repair for pathology related to their thoracic endograft, in whom secondary endovascular procedures were not possible.

\section{MATERIALS AND METHODS}

The data for the study were obtained from a prospectively maintained database. From November 1998 to October 2010, 186 patients underwent TEVAR for various diseases of the thoracic aorta. Among them, 7 patients $(3.7 \%)$ with life-threatening complications of failed TEVAR underwent open surgical repair after failed thoracic endovascular aortic repair (Table 1).

There were 5 men and 2 women, with a mean age $64.3 \pm 19$ years (range, $27-75$ years). Initial endovascular stent-grafts were placed in these patients for thoracic aortic or aortic arch aneurysm, traumatic rupture of the thoracic aorta, or aortobronchial fistulae. The indications of stent-graft explantation and open repair included device collapse, stent-graft infection, aortoesophageal fistula, aneurysm enlargement without endoleak, and RTAD.

\section{Retrograde Type A Dissection}

Three patients had an RTAD after exclusion of an aortic arch aneurysm (Figure 1). A 76-year-old woman (patient 1) was referred to the Arnaud de Villeneuve Hospital with a right aberrant retroesophageal subclavian artery associated with a 60-mm aortic arch aneurysm. Despite recent advances, reconstruction of the aortic arch remains a challenge, particularly in elderly patients. Therefore, hybrid surgery was performed. Aortic arch debranching 


\section{Abbreviations and Acronyms \\ $\mathrm{CT}=$ computed tomography \\ LCCA $=$ left common carotid artery \\ RTAD $=$ retrograde type A dissection \\ TEVAR $=$ thoracic endovascular aortic aneurysm repair}

was performed via a median sternotomy. After systemic heparinization and partial crossclamping of the ascending aorta, a proximal end-to-side anastomosis was performed with a prosthetic $10-\mathrm{mm}$ graft, followed by 2 endto-side anastomoses to the brachiocephalic trunk and the left common carotid artery (LCCA) and an end-to-end anastomosis to the left subclavian artery. Exclusion of the aneurysm was achieved by the deployment of a TAG stent-graft (WL Gore \& Associates, Inc, Flagstaff, Ariz) during the same operation. Intraoperative angiography did not show retrograde aortic dissection. On postoperative day 4 , chest pain conducted to diagnose acute RTAD. During surgical conversion, exploration of the ascending aorta disclosed an intimal tear located around the ascending aorta anastomosis. The lateral crossclamping of the ascending aorta during the debranching procedure seemed to be the cause of the ascending aortic dissection.

A 60-year-old woman (patient 2) presented with a voluminous aneurysm of a right aortic arch with a retroesophageal course. The first aortic branch was the LCCA. The left subclavian artery originated as the last branch with an anomalous retroesophageal course associated with Kommerell's diverticulum. The presence of a right aortic arch aneurysm makes open treatment difficult because of the pattern of the supra-aortic vessels, the location of the descending aorta, and the relation between the esophagus and the aortic arch. Endovascular repair has been successfully proposed as an alternative in the treatment of this entity. ${ }^{1}$ A 1-stage hybrid surgical and endovascular procedure was performed: hemiarch debranching with a left carotid-subclavian transposition and carotid-to-carotid crossover bypass. In the same operation, a C-TAG stent-graft (WL Gore \& Associates, Inc) was positioned with an adequate landing zone just distal to the LCCA. The patient was asymptomatic, but the postoperative computed tomography (CT) scan showed an acute RTAD. The retrograde dissection was probably due to a retrograde arterial wall dissection involving the LCCA, induced by retrograde carotid arteriography.
A 58-year-old man (patient 3) was referred to our department for a ruptured degenerative aortic arch aneurysm. An emergency hybrid procedure was performed with median sternotomy supra-aortic debranching and endovascular stent-grafting (Valiant stent-graft, Medtronic Inc, Minneapolis, Minn). CT on postoperative day 6 showed an RTAD with an intimal tear at the tip of the proximal bare spring. The proximal bare spring may play a role in the development of RTAD.

\section{Stent-Graft Collapse}

A 27-year-old patient (patient 4) presented with a traumatic rupture of the thoracic aorta and underwent emergency thoracic stent-graft placement. Because of the severity of the associated lesions in the patient (intracranial hematoma, spleen and left kidney contusions), despite an acute aortic arch angulation (100 degrees), endovascular repair was proposed as first-line treatment. Stent-graft collapse was observed 30 days after implantation by a pseudocoarctation syndrome leading to functional renal failure.

\section{Aneurysm Enlargement Without Endoleak}

A 75-year-old patient (patient 5) underwent endovascular aortic repair for a 7-cm descending thoracic aortic aneurysm. Twelve months later, an additional distal thoracic stent-graft was placed for a distal type I endoleak in the patient. Eighteen months later, a control CT scan showed an aneurysm enlargement without evident endoleak. The patient required graft explantation of the stent-graft and open thoracic aneurysm repair.

\section{Aortoesophageal Fistula}

An 86-year-old woman (patient 6), with a history of coronary insufficiency, had undergone endovascular repair of a ruptured descending thoracic aortic aneurysm 18 months previously. She was admitted to our hospital for backache and anorexia. An emergency CT scan disclosed a large aortoesophageal fistula. Despite her age, the absence of cardiac or pulmonary dysfunction prompted us to propose surgical conversion. She required emergency graft explantation of the infected stent-graft, open thoracic aneurysm repair, and total esophagectomy.

\section{Thoracic Stent-Graft Infection}

A 75-year-old man (patient 7), with a history of chronic renal failure and coronary insufficiency, previously underwent a combined open repair of a descending thoracic aneurysm and non-small cell lung cancer (left upper lung). One year later, he presented with acute respiratory failure and

TABLE 1. Demographic data and survey of thoracic conversion procedures

\begin{tabular}{|c|c|c|c|c|c|c|c|c|}
\hline Patient & Sex & Age, $y$ & Aortic pathology & Stent graft & Failure & $\begin{array}{c}\text { Time to } \\
\text { conversion }\end{array}$ & Conversion & Outcome \\
\hline 1 & $\mathrm{~F}$ & 76 & Aortic arch aneurysm & $\begin{array}{l}\text { TAG (WL Gore \& } \\
\text { Associates, Inc, } \\
\text { Flagstaff, Ariz) }\end{array}$ & $\begin{array}{l}\text { Retrograde type A } \\
\text { dissection }\end{array}$ & $4 \mathrm{~d}$ & $\begin{array}{l}\text { Supracoronary ascending } \\
\text { aorta replacement }\end{array}$ & Discharged \\
\hline 2 & $\mathrm{~F}$ & 60 & Aortic arch aneurysm & C-TAG & $\begin{array}{l}\text { Retrograde type A } \\
\text { dissection }\end{array}$ & $8 \mathrm{~d}$ & $\begin{array}{l}\text { Supracoronary ascending } \\
\text { aorta replacement }\end{array}$ & Discharged \\
\hline 3 & M & 58 & $\begin{array}{l}\text { Ruptured aortic arch } \\
\text { aneurysm }\end{array}$ & $\begin{array}{l}\text { Valiant (Medtronic Inc, } \\
\text { Minneapolis, Minn) }\end{array}$ & $\begin{array}{l}\text { Retrograde type A } \\
\text { dissection }\end{array}$ & $6 \mathrm{~d}$ & Bentall procedure & Discharged \\
\hline 4 & M & 27 & $\begin{array}{l}\text { Traumatic transection } \\
\text { of the aorta }\end{array}$ & TAG & Stent-graft collapse & $30 \mathrm{~d}$ & $\begin{array}{l}\text { Descending aortic } \\
\text { replacement }\end{array}$ & Discharged \\
\hline 5 & M & 67 & $\begin{array}{l}\text { Descending thoracic } \\
\text { aneurysm }\end{array}$ & Valiant and TAG & $\begin{array}{l}\text { Aneurysm } \\
\text { enlargement }\end{array}$ & $20 \mathrm{mo}$ & $\begin{array}{l}\text { Descending aortic } \\
\text { replacement }\end{array}$ & Discharged \\
\hline 6 & $\mathrm{~F}$ & 86 & Ruptured TAA & TAG & $\begin{array}{l}\text { Aortoesophageal } \\
\text { fistula }\end{array}$ & $18 \mathrm{mo}$ & $\begin{array}{l}\text { Descending aortic } \\
\text { replacement and } \\
\text { esophagectomy }\end{array}$ & Died \\
\hline 7 & M & 75 & Aortobronchial fistulae & TAG & Stent-graft infection & $60 \mathrm{~d}$ & $\begin{array}{l}\text { Ascending to supraceliac } \\
\text { abdominal aorta bypass }\end{array}$ & Died \\
\hline
\end{tabular}




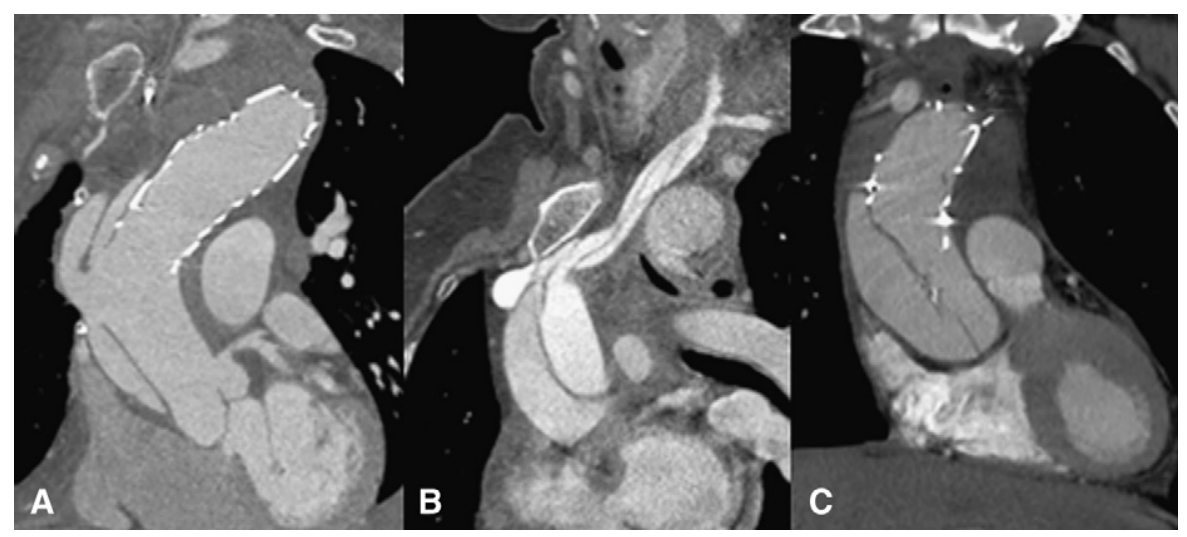

FIGURE 1. Acute retrograde type A aortic dissection after aortic arch debranching. A, Patient 1. B, Patient 2. C, Patient 3.

hemoptysis. An emergency CT scan showed an aortobronchial fistulae. In such a situation, and because of this patient's serious comorbidities, reoperation of the descending aorta is a technically complex procedure. He was treated by the deployment of a thoracic stent-graft and was discharged with long-term antibiotics. Two months later, he presented with hematemesis and evidence of mediastinitis with thoracic stent-graft infection. He underwent thoracic aortic ligation, extra-anatomic bypass (ascending to supraceliac abdominal aorta bypass), and stent-graft explantation.

\section{Surgical Procedures}

Retrograde type A dissection. In patient 1, after redo sternotomy, cardiopulmonary bypass (CPB) was established between the $10-\mathrm{mm}$ graft and the supra-aortic trunks and right atrium. Cerebral protection was achieved by means of selective antegrade perfusion via the arterial cannula introduced in the 10 -mm graft. During circulatory arrest with mild hypothermia (vesical temperature at $28^{\circ} \mathrm{C}$ ), the ascending aorta was transected disclosing an intimal tear located around the ascending aorta anastomosis. The proximal covered spring of the stent-graft was removed. The distal part of the graft was sutured to the proximal end of the stent-graft. Distal perfusion was reestablished through an inflated Foley catheter placed in the distal aortic arch. The supracoronary ascending aorta was replaced with a $30-\mathrm{mm}$ graft with reimplantation of the $10-\mathrm{mm}$ prothesis to the supra-aortic trunks. The proximal anastomosis was performed at the level of the sinotubular junction.

In patient $2, \mathrm{CPB}$ was established between the right femoral artery and the right atrium. During cooling, the nondissected right common carotid artery was cannulated. The LCCA was crossclamped, and anterograde cerebral perfusion was achieved through the cannula introduced via the right common carotid artery. During circulatory arrest, the ascending aorta was transected. The supracoronary ascending aorta was replaced with a $30-\mathrm{mm}$ graft. The distal part of the graft was sutured to the proximal end of the stent-graft. An end-to-side anastomosis with the ascending aortic graft and a 10-mm graft was performed. The $10-\mathrm{mm}$ graft was cannulated, allowing distal perfusion and global warming. The proximal aortic anastomosis was performed. LCCA ligature was performed, and the 10-mm graft was anastomosed to the non-dissected LCCA just before the carotid-to-carotid bypass.

In patient $3, \mathrm{CPB}$ was instituted between the right subclavian artery, the right femoral artery, and a bicaval cannulation. A Bentall procedure was performed. During a brief circulatory arrest (2 minutes), an endovascular occlusive balloon (Coda; Cook Inc, Bloomington, Ind) was inserted in the descending aorta, allowing a satisfactory distal perfusion $(2.5 \mathrm{~L} / \mathrm{min})$ by the femoral cannula during the distal anastomosis.

Stent-graft collapse/aneurysm enlargement without endoleak. Via a left thoracotomy, on femoro-femoral cardiopulmonary bypass, the stent-graft was explanted and a descending thoracic aneurysm repair was performed using an interposition tube graft.
Aortoesophageal fistula. Via a left thoracotomy, on femorofemoral cardiopulmonary bypass, the stent-graft was explanted and an interposition aortic tube graft repair (silver-coated polyester graft) of the descending thoracic aorta was performed. The area of esophageal disruption exceeded $3 \mathrm{~cm}$ in length, so no attempts were made to reconstruct the esophagus. A total esophagectomy was performed in the same operation, stapling the esophagus at the level of the diaphragm (Figure 2).

Thoracic stent-graft infection. The patient underwent a staged procedure. In the first stage, via a sterno-laparotomy approach, an ascending to supraceliac abdominal aortic bypass and a ascending aorta to LCCA bypass were performed. During the same operation, the aortic arch just above the LCCA was ligated. Two days later, via a redo-thoracotomy, the stent-graft and the entire descending thoracic aorta were removed because they were associated with mediastinal drainage.

\section{RESULTS \\ Postoperative Course}

Thirty-day mortality was $28 \%$. There were no cases of any neurologic complications, including stroke, paresis, or paralysis.

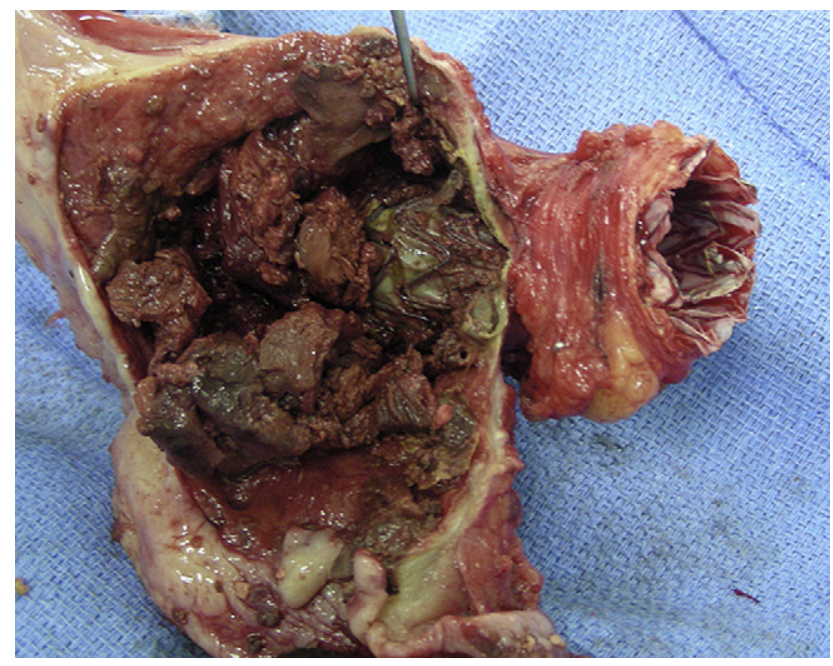

FIGURE 2. Aortoesophageal fistula after TEVAR. En bloc resection of the esophagus and descending thoracic aorta showing a large aortoesophageal fistula. 


\section{Retrograde Type A Dissection (Patients 1, 2, and 3)}

The postoperative course was uneventful for the 3 patients, except a postoperative sternitis successfully treated by prolonged intravenous antibiotic therapy in the third patient.

\section{Stent-Graft Collapse (Patient 4)/Aneurysm Enlargement Without Endoleak (Patient 5)}

The postoperative course was uneventful. Patients treated for stent-graft collapse and aneurysm enlargement were discharged on postoperative days 8 and 12 , respectively.

\section{Aortoesophageal Fistula (Patient 6)}

The patient died of early acute respiratory distress syndrome 5 days postsurgery.

\section{Thoracic Stent-Graft Infection (Patient 7)}

The patient died of multiorgan failure 7 days postsurgery.

\section{Follow-up}

During a mean follow-up of 21.4 months (range, 2-60 months), all surviving patients were in good clinical condition.

\section{DISCUSSION}

The current series of 7 surgical conversions after thoracic endograft placement provides additional patient cohorts to the literature regarding the utility of surgical treatment to salvage failed endovascular thoracic aortic procedures. The conversion rate from our own monocentric cohort of 176 patients was $3.9 \%$. This was similar to conversion rates of $3.8 \%$ as recently reported in the Talent Thoracic Registry among 457 patients treated in various European institutions. $^{2}$

RTAD after thoracic endovascular aortic repair has been reported, ranging between $1 \%$ and $6.8 \%$ with a high risk of mortality. ${ }^{3}$ RTAD occurs not only during surgery but also days, weeks, or months after surgery. There are many possible mechanisms causing intimal tear during surgery, including wire and sheath manipulation in the aortic arch, lateral crossclamping of the ascending aorta, and impact forces during stent-graft deployment. However, the influence of the different stent-grafts on intimal injury remains uncertain. The proximal bare spring may play a role in the development of RTAD. Balloon remodeling to match the curved aortic arch and routine oversizing more than $20 \%$ of the stent-graft may contribute to the development of RTAD. Fragility of the aortic wall, as presented in the study by Dong and colleagues ${ }^{3}$ (with 3 cases of RTAD involving Marfan syndrome), may contribute to the development of RTAD. As suggested in this short series, aortic arch malformation also can contribute to the development of RTAD. In our experience, 2 patients with aberrant subclavian arteries underwent a supra-aortic debranching procedure. These 2 patients had an RTAD. Aberrant subclavian artery weakens the aortic wall, leading to higher wall stress, which can induce aortic dilatation and aneurysm formation, and eventually favor the occurrence of an aortic dissection. This subset of patients with aberrant subclavian arteries or aortic arch malformation seem to have a high risk of RTAD; therefore, for this population, the hybrid procedure should be avoided. As previously reported, ${ }^{4}$ the stent-graft should not be removed to avoid worsening the injured aortic wall. The distal anastomosis should also be performed by connecting the ascending aortic graft to the stent-graft, taking deep bites in the surrounding aortic wall after the removal the proximal spring. The modalities of cerebral protection are simplified because of the prior transposition of the supra-aortic trunks, allowing a satisfactory anterograde cerebral perfusion by cannulating the graft to the supra-aortic trunks. For circulatory arrest during distal anastomosis, the most attractive strategy was performed in patient 3 . The rigid structure of the stent-graft allowed efficient occlusion of the aorta with an endovascular occlusive balloon. Distal perfusion was achieved via the femoral artery with satisfactory flow, thus avoiding prolonged deep hypothermia and complications associated with excessive cooling.

We previously studied and reported ${ }^{5}$ factors leading to thoracic stent-graft collapse. In our patients, stent-graft collapse after TEVAR seems to be related to poor apposition of the stent-grafts in a highly angulated aortic arch or excessive stent-graft oversizing. In planning a treatment strategy, assessment of aortic arch anatomy and appropriate choice of the device may prevent such a complication. In cases of poor apposition of the proximal edge of the stent-graft to the inner curve of the aorta, close CT follow-up should be performed. Furthermore, the use of adjunctive technologies to enhance fixation of the proximal stent-graft should be considered. Improvement in stent-graft conformability and the availability of a stent-graft with a small diameter should decrease the incidence of this device-related complication.

Aortoesophageal fistula after TEVAR is a rare unusual complication of TEVAR (incidence 1.9\%). ${ }^{6}$ Conservative strategies, including broad-spectrum antibiotics, protonpump inhibitors, and enteral feeding, remain far from optimal for the management of this condition. Furthermore, conservative treatment is almost invariably a fatal outcome, justifying a more aggressive surgical strategy involving esophageal resection, effective debridement, and aortic repair. However, operative management of aortoesophageal fistula carries significant mortality, especially in patients with comorbidities and a general health status that initially precluded open surgical repair. Mortality after surgery for thoracic aortic fistulae has been shown to reach $78 \%$ in cases of secondary fistulae. ${ }^{7}$ Thoracic endovascular aortic repair of aortobronchial fistulas seems to be a viable alternative to conventional open repair with excellent shortterm results. ${ }^{8}$ However, the risk of recurrence of the 
aortobronchial fistula or of infection of the stent-graft after endovascular repair is high, and this approach should be used as bridge to definitive open repair. Stent-graft infection is rarely reported, but this is a devastating complication of aortic disease management. Patient 7 had previously undergone a combined open repair of a descending thoracic aneurysm and left upper lobectomy. A stent-graft had been also deployed for aortobronchial fistulae. In situ reconstruction with prosthetic bypass has been successfully performed, ${ }^{8}$ but this carries the potential for graft infection and new fistulization. Furthermore, obtaining adequate exposure with conventional proximal and distal vascular control may place the patient at risk for life-threatening hemorrhage. Cryopreserved homografts represent a viable option for in situ aortic reconstruction, ${ }^{9}$ although they are susceptible to infection, and long-term secondary degeneration has been described in early and late outcomes. We thought that access to the aorta would be particularly demanding because of dense adhesions and mediastinitis with a high risk of adjacent organ lesions and significant blood loss. In addition, we thought that extra-anatomic bypass arising from the ascending aorta (ventral aorta) would reduce the risk of graft infection. ${ }^{10}$ However, in our experience, patient 7 died of multiorgan failure.

Persistent aneurysm enlargement after an initial successful endovascular thoracic repair was also observed in $1 \mathrm{pa}-$ tient (patient 5). Repeated CT scan showed no endoleak. Because of the progressive aneurysm enlargement, a surgical conversion was necessary to remove the endograft and reconstruct the aorta. During surgery, on opening the aneurysm sac, no visible blood flow was detected from the endograft attachment site or intercostal arteries. Furthermore, stent-graft explantation showed no devicerelated failure. This failure of exclusion likely represents the presence of pressurized aneurysm or endotension. ${ }^{11}$

Surgical repair of a failed thoracic stent-graft is a complex procedure often requiring extracorporeal circulation and adjunctive modalities to provide organ protection. With the increasing use of TEVAR, more patients will present with emergency indications for surgical correction; thus, knowledge regarding TEVAR conversion is mandatory. Zipfel and colleagues $^{12}$ reported 6 patients requiring conversion to open repair after TEVAR, 3 of whom required explantation immediately after deployment during the same operation, and 3 of whom required repair before discharge (RTAD, aortic rupture, and failed access): One patient died, 1 patient had an extended hospital stay, and 1 patient was successfully discharged. Patients treated more electively with delayed conversion were all discharged. In our series, none of the patients underwent immediate intraoperative conversion, and except for those with stent-graft infection, all patients were discharged. The worse results were associated with the management of thoracic stent-graft infection. However, patients 6 and 7 had comorbidities and a general health sta- tus that initially precluded open surgical repair and presented impaired general health status due to severe sepsis.

\section{CONCLUSIONS}

Our experience suggests that in some clinical or anatomic situations, caution should be recommended when offering endovascular procedures to patients with thoracic aortic diseases. First, aberrant subclavian arteries or aortic arch malformation and stent-grafts with proximal bare-stent seem to favor the occurrence of retrograde ascending aortic dissection. Second, endovascular aortic repair of aortobronchial fistulas is associated with a high risk of recurrence of the aortobronchial fistula or infection and should be used as a bridge to definitive open repair. Third, patients with acute aortic arch angulation or a small aortic diameter, frequently observed in young patients with acute traumatic transection of the aorta, open surgical treatment should be discussed to avoid thoracic stent-graft collapse. Surgical repair of a failed thoracic stent-graft is a complex procedure. Open conversion can be performed with encouraging results by a team experienced in the management of thoracic aortic diseases. With the increasing use of TEVAR, more patients will present with indications for surgical correction; thus, knowledge regarding TEVAR conversion is mandatory given that the procedure is being performed for more challenging anatomy and broader indications.

\section{References}

1. Okada K, Sueda T, Orihashi K, Watari M, Naito A. Endovascular stent-graft repair for thoracic aortic aneurysm associated with right-sided aortic arch. J Thorac Cardiovasc Surg. 2001;122:185-6.

2. Ehrlich MP, Nienaber CA, Rousseau H, Beregi JP, Piquet P, Schepens M, et al. Shortterm conversion to open surgery after endovascular stent-grafting of the thoracic aorta: the Talent thoracic registry. J Thorac Cardiovasc Surg. 2008;135:1322-6.

3. Dong ZH, Fu WG, Wang YQ, Guo da Q, Xu X, Ji Y, et al. Retrograde type A aortic dissection after endovascular stent-graft placement for treatment of type B dissection. Circulation. 2009;119:735-41.

4. Savini C, Casselman F, Ergenoglu MU, Degrieck I, Van Praet F, DeGeest R, et al Surgical management of progression to type A dissection from an intramural hematoma previously treated with endovascular stent-graft placement. $J$ Thorac Cardiovasc Surg. 2004;128:773-5.

5. Canaud L, Alric P, Desgranges P, Marzelle J, Marty-Ané C, Becquemin JP. Factors favoring stent-graft collapse after thoracic endovascular aortic repair J Thorac Cardiovasc Surg. 2010;139:1153-7.

6. Eggebrecht H, Mehta RH, Dechene A, Tsagakis K, Kühl H, Huptas S, et al. Aortoesophageal fistula after thoracic aortic stent-graft placement: a rare but catastrophic complication of a novel emerging technique. JACC Cardiovasc Interv. 2009;2:570-6

7. Pipinos II, Reddy DJ. Secondary aortoesophageal fistulae. Ann Vasc Surg. 1999; 13:649-52.

8. Riesenman PJ, Brooks JD, Farber MA. Thoracic endovascular aortic repair of aortobronchial fistulas. J Vasc Surg. 2009;50:992-8.

9. Kieffer E, Chiche L, Gomes D. Aortoesophageal fistula: value of in situ aortic allograft replacement. Ann Surg. 2003;238:283-90.

10. Madan AK, Santora TA, Disesa VJ. Extra-anatomic bypass grafting for aortoesophageal fistula: a logical operation. J Vasc Surg. 2000;32:1030-3.

11. Mehta M, Veith FJ, Ohki T, Lipsitz EC, Cayne NS, Darling RC 3rd. Significance of endotension, endoleak, and aneurysm pulsatility after endovascular repair. $J$ Vasc Surg. 2003;37:842-6.

12. Zipfel B, Hammerschmidt R, Krabatsch T, Buz S, Weng Hetzer R. Stent-grafting of the thoracic aorta by the cardiothoracic surgeon. Ann Thorac Surg. 2007;83 441-8. 